Joachim Nelles ${ }^{1}$

Heino Stöver ${ }^{2}$

\section{Zehn Jahre Spritzenvergabe im Gefängnis: Ein Review der bisherigen Spritzenvergabeprojekte in der Schweiz, Deutschland, Spanien und Moldawien}

\author{
Ten Years of Syringe Exchange Programmes in Prison. A Review of Syringe \\ Exchange Programmes in Switzerland, Germany, Spain and Moldavia
}

\section{Zusammenfassung}

In diesem Beitrag werden die praktischen Erfahrungen und wissenschaftlichen Erkenntnisse aus den seit 10 Jahren bestehenden Spritzenvergabeprojekten in Gefängnissen in der Schweiz, Deutschland, Spanien und Moldawien ausgewertet und diskutiert. Ausgangspunkt der Diskussion sind die vor Beginn solcher infektionsprophylaktischen Projekte formulierten Befürchtungen. Es zeigt sich, dass die Spritzenabgabe an Drogenabhängige im Gefängnis machbar ist, was Akzeptanz und Integration in den Anstaltsablauf betrifft. Die ursprünglichen Befürchtungen haben sich nicht bestätigt: Drogenkonsum steigt nicht an; i.v.-Konsum wird nicht begünstigt; Spritzen werden nicht missbräuchlich eingesetzt und die Entsorgung gebrauchter Spritzen stellt kein Problem dar. Neuinfektionen (nur in wenigen Gefängnissen untersucht) wurden nicht beobachtet. Spritzentausch unter Drogenabhängigen nahm entweder massiv ab oder blieb auf Einzelfälle beschränkt. Ein Durchbruch der Spritzenabgabe konnte allerdings bisher nirgendwo erreicht werden. In Spanien wurden alle Gefängnisse per Dekret angewiesen, drogenabhängigen Gefangenen sterile Spritzen zur Verfügung zu stellen. Diese Maßnahme wurde bis heute erst teilweise umgesetzt. Vielleicht hat dies eine Signalwirkung auf die Spritzenabgabe in anderen Ländern und Gefängnissen.

\section{Schliuisselwörter}

Schadensbegrenzung (harm reduction) - Spritzenabgabe • Gefängnis · HIV- und Hepatitisinfektion

\section{Abstract}

In this paper, we present results and conclusions of syringe exchange programmes in prisons based on 10 years experience in Germany, Spain, Switzerland and Moldova. Until now, the syringe exchange programme was introduced into 23 prisons and maintenance of syringe distribution is apparent in 20 of those prisons. From 11 prisons results from scientific evaluations are available. From the results it could be clearly demonstrated that none of the fears that arose, whenever syringe exchange schemes were planned, occurred in any project: Syringe distribution was not followed by an increase in drug intake or i.v. application, syringes were not misused, disposal of used syringes was uncomplicated and syringe sharing among drug addicts disappeared almost completely or was apparent in single cases only. In the prisons, where blood tests were performed, no seroconversion for HIV or hepatitis infection was observed. Thus feasibility of harm reduction including syringe exchange on the one hand and efficacy on the other hand was proven in general. However, despite these positive results, syringe exchange in prison is far beyond general acceptance, both on the prison level as well as on the political level. However, in Spain, a governmental decree was released in 2001 that all prisons have to provide drug addicts with sterile injection equipment. Yet, the outcome of this decree is still open and results are so far not available.

Key-words

Harm reduction - syringe distribution - prison $\cdot$ HIV- \& hepatitisinfection 


\section{Spritzenabgabe und Schadensbegrenzung}

Die Gefährlichkeit von illegalen Drogen leitet sich nicht primär von den Eigenheiten der Substanzen selbst ab, sondern ist vielmehr davon abhängig, wie mit den Substanzen umgegangen wird. „Nichts ist Gift und alles ist Gift, nur die Menge macht das Gift" - diese These von Paracelsus gilt auch heute noch uneingeschränkt. Das Drogenverbot selbst zeichnet für die meisten schwerwiegenden individuellen und gesellschaftlichen Folgen des Drogenkonsums hauptverantwortlich [1]. Obschon die Preise für illegale Drogen auf der Straße in den letzten Jahren massiv zurückgegangen sind, bleibt intravenöser Konsum von z.B. Heroin und Kokain unverändert gegenüber anderen Applikationsformen bestehen. Der Tausch von Injektionsutensilien (Nadel, Spritze, Löffel und Filter) unter Drogenkonsumenten stellt dabei die Hauptübertragungsform für so genannte „blood borne virus infections“ wie HIV und Hepatitis B und C dar, was dazu geführt hat, dass das Vorkommen dieser Infektionskrankheiten unter Drogenabhängigen um ein Vielfaches höher ist als vergleichsweise in der Normalbevölkerung [2,3].

Der Konsum illegaler Drogen ließ sich bis heute mit keiner Maßnahme wirksam verhindern. Moderne Gesellschaften zeichnen sich im Gegenteil durch eine hohe Bereitschaft an Suchtverhalten aus (für die Schweiz: Uchtenhagen in Nelles: Drogenpolitik wohin?, Gutzwiller in Nelles: Harm Reduction in Prison). Schaden zu verhindern oder zumindest $\mathrm{zu}$ begrenzen, wenn risikobehafteter Drogenkonsum selbst nicht oder nicht wirksam genug verhindert werden kann, entspricht deshalb nur einer konsequenzialistischen Ethik. Schadensbegrenzung ist dabei nicht gleichzusetzen mit Drogenpermissivität, wie dies von Kritikern allzu gerne und allzu leichtfertig behauptet wird [4].

Die wirksamste Maßnahme allein zur Begrenzung der Übertragung von HIV und Hepatitis als Folge risikobehafteten Drogenkonsums - das haben zahlreiche Studien in verschiedenen Ländern eindeutig belegt - ist die Abgabe steriler Spritzen an intravenös Drogen konsumierende süchtige Menschen [5]. Diese schadensbegrenzende Maßnahme hat sich denn auch in praktisch allen Ländern mit ernsthaftem Drogenproblem etabliert, wobei die Spritzen mehrheitlich über öffentlich aufgestellte Austauschautomaten, in Apotheken sowie in Drogenberatungs- oder Kontaktstellen erhältlich sind.

\section{Besondere Situation Gefängnis - oder was lässt die Spritzen- abgabe im Gefängnis so widersprüchlich erscheinen?}

Gefängnisse erscheinen - oberflächlich betrachtet - als Spiegelbild der Gesellschaft. Die Verzerrung dieses Bildes wird aber bei genauerer Betrachtung rasch deutlich: 95\% aller Insassen in Gefängnissen sind Männer, die meisten in jugendlichem Alter. Körperliche und psychische Krankheiten sind auffallend häufig. Die Einnahme von Medikamenten ist deutlich erhöht. Etwa die Hälfte der Insassen (in Frauengefängnissen sogar bis zu 80\%) konsumiert illegale Drogen wie Kokain und Heroin. Etwa die Hälfte dieser Insassen setzt den Konsum der Drogen auch während des Gefängnisaufenthalts fort, dann mehrheitlich intravenös appliziert. Das Vorkommen von positiven HIV- und Hepatitis-B- und C-Befunden ist um ein Vielfaches erhöht [6, 7]. Im Gefängnis wird allein schon der Besitz von Spritzen und anderen Injektionsmaterialien sanktioniert, was verdeckten Konsum und das Tauschen von Spritzen begünstigt. Zahlungsmittel für illegale Drogen sind nicht selten sexuelle Kontakte unter Insassen, aber auch teilweise zwischen Insassen und Personal. Ein Großteil der Betroffenen schützt sich dabei nicht mit Kondomen [8, 9], was die Gefahr der Übertragung von Infektionskrankheiten, vor allem HIV und Hepatitis, noch einmal begünstigt.

Die Verbreitung solcher Infektionskrankheiten im Gefängnis ist eindeutig und vielfältig belegt [10]. Der Ruf nach schadensbegrenzenden Maßnahmen, zumindest unter Wissenschaftlern, erstaunt deshalb nicht. Denn: Gefängnisse sind keine geschlossenen Systeme. Urlaube, Verlegungen, vorzeitige Entlassungen und eine zunehmende Zahl an Kurzstrafen führen zu der für moderne Gefängnisse typischen hohen Fluktuation. Auf diese Weise kommen, betrachtet man die große Zahl an Gefangenen (in industrialisierten Ländern typischerweise etwa 100 Insassen pro 100000 Einwohner), beachtliche Anteile der Bevölkerung mit der Gefängniswelt - und damit mit HIV/AIDS und Hepatitis - in enge Berührung. Doch ungeachtet dessen blieben mit Ausnahme weniger Pilotprojekte die Gefängnistore für schadensbegrenzende Maßnahmen wie der Abgabe von sterilen Spritzen fest verschlossen.

Wie ist das zu erklären? Drogenabhängige sind wegen oder im Zusammenhang mit ihrer Sucht im Gefängnis. Die Abgabe steriler Spritzen wird nicht als Ausdruck einer konsequenzialistischen Ethik verstanden, sondern vielmehr als Unterstützung des Konsums illegaler Drogen, wobei der Anspruch auf einen drogenfreien Raum im Gefängnis als geschlossener und kontrollierter Institution noch stärker zum Ausdruck kommt. Hieraus ist auch ableitbar, dass die meisten Gefängnisse ein ernsthaftes Drogenproblem negieren und schadensbegrenzende Maßnahmen allein schon deshalb als überflüssig ablehnen. Drogenkonsum mit allen möglichen Mitteln zu verhindern suchen, vorkommenden Drogenkonsum entsprechend zu sanktionieren und gleichzeitig Spritzen zur Schadensbegrenzung zur Verfügung zu stellen - das wird als „nicht vollführbarer Spagat“ empfunden. Widerstände gegenüber der Spritzenabgabe äußern sich in Form folgender Befürchtungen und Behauptungen: Spritzenabgabe stimuliere zum Drogenkonsum (Neueinsteiger, Wiedereinsteiger), begünstige die mit mehr Risiken behaftete intravenöse Applikationsform, fördere den Missbrauch von Spritzen z. B. als Waffe gegenüber Bediensteten oder Mitgefangenen, führe zu schlecht entsorgten Spritzen, was mit einem erhöhten Verletzungs- und Ansteckungsrisiko einhergehe, und hätte letztlich keinen Einfluss auf die Prävalenz von HIV- und Hepatitisinfektionen. Es sei auch fraglich, ob Spritzentausch unter drogenabhängigen Insassen wirklich verhindert oder vermindert werden könne und ob Drogenabhängige zu Verhaltensänderungen in der Lage seien.

\section{Das Äquivalenzprinzip}

Europarat und WHO haben schon im Jahr 1993 in verschiedenen Resolutionen die Forderung aufgestellt, dass in Gefängnissen die gleichen präventiven und schadensbegrenzenden Maßnahmen einzuführen seien wie in der freien Gesellschaft, d.h., dass z.B. in jenen Ländern, in welchen Drogenabhängigen Spritzen und Kondome zur Verfügung gestellt werden, diese Maßnahmen auch im Gefängnis einzuführen seien. Diese Forderung nach dem Äquivalenzprinzip, d. h. der Gleichstellung schadensbegrenzender Maßnahmen aus gesundheitspolitischer und ethischer 
Sicht innerhalb und außerhalb von Gefängnissen, konnte der Spritzenabgabe als Teil eines schadenbegrenzenden Angebots im Strafvollzug allerdings bis heute nirgendwo zum Durchbruch verhelfen, war aber hilfreich bei der politischen Argumentation im Vorfeld verschiedener geplanter und wissenschaftlich begleiteter Pilotprojekte [11 - 13].

\section{Spritzenabgabeprojekte in Europa und Südosteuropa}

\section{Schweiz}

Weltweit erstmalig bewilligten die politischen Instanzen des Kantons Solothurn 1992 offiziell die Abgabe steriler Spritzen an drogenabhängige Insassen im Gefängnis von Oberschöngrün durch den Gefängnisarzt Dr. Probst, anstelle diesen zu entlassen - denn Dr. Probst hatte zuvor in seiner Sprechstunde regelmäßig sterile Spritzen ohne Erlaubnis an Insassen abgegeben. Die offizielle Abgabe steriler Spritzen im Gefängnis nahm so als „medizinischer Ungehorsam" ihren Anfang [14]. 1994 folgte in den Anstalten von Hindelbank (Kanton Bern) das erste wissenschaftlich begleitete Präventionsprojekt mit der Abgabe steriler Spritzen via 1:1-Austauschautomaten [15]. Mit den Projekten in den Gefängnissen von Realta (Kanton Graubünden) und Saxerriet (Kanton St. Gallen) gab es noch zwei weitere wissenschaftlich begleitete Spritzenabgabeprojekte in der Schweiz $[9,16] .1998$ wurden die Gefängnisse Thorberg und Witzwil im Kanton Bern per Dekret durch die Polizei- und Militärdirektion verpflichtet, drogenabhängigen Insassen auf Verlangen sterile Spritzen zur Verfügung zu stellen. Basis hierfür bildeten die positiven Ergebnisse der Projekte in Hindelbank und Realta sowie ein Rechtsgutachten des Schweizerischen Bundesamtes für Justiz, welches die Frage der Verpflichtung von Vollzugsinstitutionen zur Abgabe von sterilen Injektionsutensilien bejahte [17].

\section{Deutschland}

Nach schweizerischem Vorbild wurden in Niedersachsen im April bzw. Juli 1996 in der JVA für Frauen in Vechta und in der JVA für Männer in Lingen/Abt. Groß Hesepe die ersten Spritzenabgabeprojekte eingeführt [18, 19]. 1996 folgten Spritzenabgabeprojekte in einer Anstalt des offenen Vollzugs in Hamburg [20] und zwei in Berlin [21] sowie zwei weitere in Hamburg [22]. Alle Projekte wurden wissenschaftlich begleitet. Unterschiede gab es vor allem in den Abgabemodi, konzeptionellen Zielsetzungen, praktischen Umsetzungen sowie dem Einbezug und der Rolle externer Gruppen [23]. Auf politischen Beschluss hin wurden die drei Hamburger Projekte im Frühjahr 2002 wieder eingestellt.

\section{Spanien}

In Spanien bestehen zurzeit in elf Gefängnissen Spritzenaustauschprogramme. Das erste Pilotprojekt begann 1997 auf Beschluss des baskischen Parlaments in der Nähe von Bilbao (Basauri). Ein zweites Projekt folgte 1998 auf Weisung lokaler Instanzen in Pamplona. Nach der positiven Evaluation der beiden Pilotprogramme wurden neun weitere Projekte lanciert: zwei 1999, fünf im Jahr 2000 und zwei im Jahr 2001. Zwei weitere Spritzenabgabeprojekte sollen im Sommer 2002 eingeführt werden. Im Juni 2001 erging eine Weisung des Generaldirektorats des spanischen Justizvollzugssystems, wonach alle 68 Anstalten sukzessive Spritzenvergabeprojekte einführen müssen [24]

\section{Moldawien}

Die HIV-Krise in Osteuropa hat in diesem Land dazu geführt, pragmatische Wege zu gehen. In einem geschlossenen Gefängnis mit 800 erwachsenen Insassen in Moldawien existiert seit 1999 ein Spritzenvergabeprojekt, das die Spritzenvergabe über Peers und durch die medizinische Abteilung durchführt [25].

\section{Gesamtübersicht}

In Tab. 1 sind alle bisherigen Spritzenabgabeprojekte und die wichtigsten Details zu den Projekten aufgelistet. In der Schweiz, in Deutschland, Spanien und Moldawien wurden bis heute offiziell in insgesamt 23 Gefängnissen Spritzen abgegeben. Außer in den drei Hamburger Anstalten blieb die Spritzenabgabe auch nach Abschluss des Modellversuchs als festes Angebot bestehen. Wissenschaftliche Untersuchungen fanden in zwölf Gefängnissen statt.

\section{Ergebnisse}

Von insgesamt elf Projekten liegen heute wissenschaftlich erhärtete Resultate der Spritzenabgabe vor. Von besonderem Interesse waren bei den meisten Projekten folgende Fragen: Steigt der Drogenkonsum an? Wird vermehrt intravenös konsumiert? Werden Spritzen missbräuchlich verwendet? Werden die Spritzen ordentlich entsorgt? Nimmt der Tausch von Spritzen unter drogenabhängigen Insassen ab? Verändert sich die Prävalenz von HIV/AIDS und Hepatitis unter der Insassenpopulation?

In Tab. 2 sind die wichtigsten Kerngrößen im Zusammenhang mit den zentralen Fragen aus verschiedenen Forschungsprojekten nebeneinander dargestellt. Fazit: Keine der im Vorfeld verschiedener Projekte erhobenen Befürchtungen hat sich bisher bestätigt. Der Drogenkonsum stieg in keiner der untersuchten Anstalten an. In Hindelbank und Realta nahm er über die Zeit sogar signifikant ab. Auch der i.v.-Konsum stieg nirgendwo an. Es fand, mit wenigen Ausnahmen, auch kein Wechsel von weniger risikobeladenen Konsumformen zum i.v-Konsum statt (vgl. hierzu exemplarisch Abb.1). Es wurden auch, mit ganz wenigen Ausnahmen, keine Neueinsteiger in den Drogenkonsum beobachtet. Spritzen werden von den Insassen zum Konsum von Drogen bezogen. Dies war in Hindelbank und Realta daran zu messen, dass der Bezug von Spritzen signifikant anstieg, wenn Drogen vermehrt in der Anstalt verfügbar waren (vgl. hierzu Abb. 2). Der Bezug stieg auch nach der Auszahlung des monatlichen Pekuliums (Arbeitsgeld) an sowie unmittelbar nach dem Wochenende, wenn Drogen bekanntermaßen vermehrt in die Anstalt gelangen. Die Akzeptanz der Spritzenabgabe durch die Insassen ist in unterschiedlichen Vergabeformen (Hand-zu-Hand oder Austauschautomat) nicht verschieden. Spritzentausch kam praktisch nicht mehr vor oder verharrte auf tiefem Niveau und reduzierte sich auf Einzelfälle. Serokonversionen, d. h. neue HIV- oder Hepatitisinfektionen, ließen sich in den Anstalten mit entsprechender Untersuchung (Hindelbank, Vechta, Hamburg, Berlin) in den Beobachtungszeiträumen nicht nachweisen. Alle Projektresultate wurden den Publikationen zu den verschiedenen Projekten entnommen $[8,19,16,26-27]$. 
Tab. 1 Spritzenabgabeprojekte in Gefängnissen in Deutschland, Spanien, der Schweizund Moldawien

\begin{tabular}{|c|c|c|c|c|c|c|c|}
\hline Gefängnis & Ort & Größe & Beginn & Geschlecht & Vollzugsform & Vergabeform & Evaluation \\
\hline Oberschöngrün & Solothurn, Schweiz & 75 & 1992 & $\mathrm{~m}$ & halboffen & Handabgabe & nein \\
\hline Hindelbank & Hindelbank, Schweiz & 110 & 1994 & $f$ & geschlossen & Automat & ja \\
\hline Champ Dollon & Genf, Schweiz & 70 & 1996 & $m \& f$ & U-Haft & Handabgabe & nein \\
\hline Lingen I & Groß Hesepe, Deutschland & 228 & 1996 & $\mathrm{~m}$ & geschlossen & Handabgabe & ja \\
\hline Vechta & Vechta, Deutschland & 239 & 1996 & $f$ & geschlossen \& U-Haft & Automat & ja \\
\hline Vierlande & Hamburg, Deutschland & 319 & 1996 & $\mathrm{~m}$ & offen & $\begin{array}{l}\text { Automat } \\
\text { Handabgabe }\end{array}$ & ja \\
\hline Basauri $^{1}$ & Vizcaya, Spanien & 250 & 1997 & $\mathrm{~m}$ & halboffen & & ja \\
\hline Realta & Graubünden, Schweiz & 100 & 1997 & $\mathrm{~m}$ & halboffen & Automat & ja \\
\hline Lehrter Straße & Berlin, Deutschland & 100 & 1998 & $\mathrm{~m}$ & geschlossen & Automat & ja \\
\hline Lichtenberg & Berlin, Deutschland & 50 & 1998 & $f$ & geschlossen & Automat & ja \\
\hline Thorberg & Krauchthal, Schweiz & 185 & 1998 & $\mathrm{~m}$ & geschlossen & Handabgabe & nein \\
\hline Witzwil & Witzwil, Schweiz & 180 & 1998 & $\mathrm{~m}$ & halboffen & Handabgabe & nein \\
\hline Am Hasenberge & Hamburg, Deutschland & 494 & 2000 & $\mathrm{~m}$ & geschlossen & Handabgabe & ja \\
\hline Hannöversand & Hamburg, Deutschland & 46 & 2000 & $f$ & offen \& geschlossen & Handabgabe & ja \\
\hline Saxerriet & Salez, Schweiz & 110 & 2000 & $\mathrm{~m}$ & offen & Automat & ja \\
\hline Branesti „No. 18“ & Moldawien & 800 & 1999 & $\mathrm{~m}$ & geschlossen & Handabgabe & nein \\
\hline
\end{tabular}

${ }^{1}$ Inzwischen sind in elf Gefängnissen in Spanien Spritzenumtauschprojekte eingeführt worden.

Tab. 2 Resultate aus wissenschaftlich untersuchten Spritzenabgabeprojekten

\begin{tabular}{lllllll}
\hline Gefängnis & Drogenkonsum & i.-v.-Konsum & $\begin{array}{l}\text { Missbrauch von } \\
\text { Spritzen }\end{array}$ & Spritzenentsorgung & Spritzentausch & $\begin{array}{l}\text { Prävalenz von } \\
\text { HIV/Hepatitis }\end{array}$ \\
\hline Am Hasenberge & kein Anstieg & kein Anstieg & nie & unproblematisch & stark vermindert & ohne Untersuchung \\
\hline Basauri ${ }^{3}$ & kein Anstieg & kein Anstieg & nie & unproblematisch & keine Information & ohne Untersuchung \\
\hline Hannöversand & kein Anstieg & kein Anstieg & nie & unproblematisch & stark vermindert & ohne Untersuchung \\
\hline Hindelbank & Abnahme & kein Anstieg & nie & unproblematisch & stark vermindert & kein Anstieg \\
\hline Lehrter Straße & kein Anstieg & kein Anstieg & nie & unproblematisch & stark vermindert & kein Anstieg ${ }^{3}$ \\
\hline Lichtenberg & kein Anstieg & kein Anstieg & nie & unproblematisch & stark vermindert & kein Anstieg \\
\hline Lingen I & kein Anstieg & kein Anstieg & nie & unproblematisch & stark vermindert & kein Anstieg \\
\hline Realta & Abnahme & kein Anstieg & nie & unproblematisch & Einzelfälle & ohne Untersuchung \\
\hline Saxerriet & keine Aussage ${ }^{1}$ & keine Aussage & nie & unproblematisch & keine Aussage ${ }^{1}$ & ohne Untersuchung \\
\hline Vechta & kein Anstieg & kein Anstieg & nie & unproblematisch & stark vermindert & kein Anstieg \\
Vierlande & kein Anstieg & kein Anstieg & nie ${ }^{2}$ & unproblematisch ${ }^{2}$ & unverändert & ohne Untersuchung \\
\hline
\end{tabular}

${ }^{1}$ Wissenschaftliche Auswertungen waren in bestimmten Bereichen nur für die Untersuchung nach Beginn der Spritzenabgabe möglich.

2 Am Anfang haben Insassen allerdings vorübergehend als Reaktion auf Drogendurchsuchung beim Spritzenbezug Automaten beschädigt und sterile Spritzen herumgestreut.

${ }^{3}$ Es trat eine (vermutlich in der Anstalt durch Infektion ausgelöste) Hepatitis-C-Serokonversion auf.

\section{Diskussion}

Spritzenumtauschprojekte lassen sich - das haben die bisherigen Erfahrungen gezeigt - ohne große Störungen in den Arbeitsablauf einer Anstalt integrieren. Sie tangieren die Beziehungen zwischen Vollzugsbediensteten, drogenabhängigen und nicht drogenabhängigen Gefangenen, indem Drogenkonsum und Infektionsprophylaxe zum Dauerthema werden. Das kann sich positiv auf das Konsumverhalten von Insassen auswirken. So konnte in Hindelbank mittels Multivarianzanalyse aufgezeigt werden, dass Insassinnen, die vor dem Gefängnisaufenthalt bereits Drogen konsumiert hatten, vermehrt auf Drogenkonsum im Gefängnis verzichteten, je länger das Projekt bereits in Hindelbank verankert war (40\% der Varianz des Drogenkonsums ließen sich so erklären; Beobachtungszeitraum zwei Jahre) [28].
Spritzenaustauschprojekte lassen Widersprüche im vollzuglichen Umgang mit Drogenkonsum deutlicher hervortreten. Die Auseinandersetzung hiermit vollzieht sich im Spannungsfeld zwischen Kontrollauftrag und gesundheitsorientierten Hilfeleistungen. Begleitende Präventions- und Informationsangebote für die Bediensteten als auch für die Inhaftierten tragen zur Verankerung und zum Erfolg der Infektionsprophylaxe in den Anstaltsalltag bei. Idealerweise sind solche Angebote auf die jeweilige Zielgruppe zugeschnitten und somit möglichst lebensweltnah. Angebote externer Anbieter erscheinen dabei besonders sinnvoll, da diese glaubwürdiger sind, eindeutigere Botschaften vermitteln und mehr Verständnis für die Zielgruppe aufweisen. Auf diese Weise lassen sich auch Präventions- und Vollzugsaufgaben am einfachsten voneinander trennen. Werden Vollzugs-, Präventionsund gar Evaluationsaufgaben miteinander vermischt (wie z.B. in Saxerriet versucht), muss mit erheblichen Widerständen von allen 


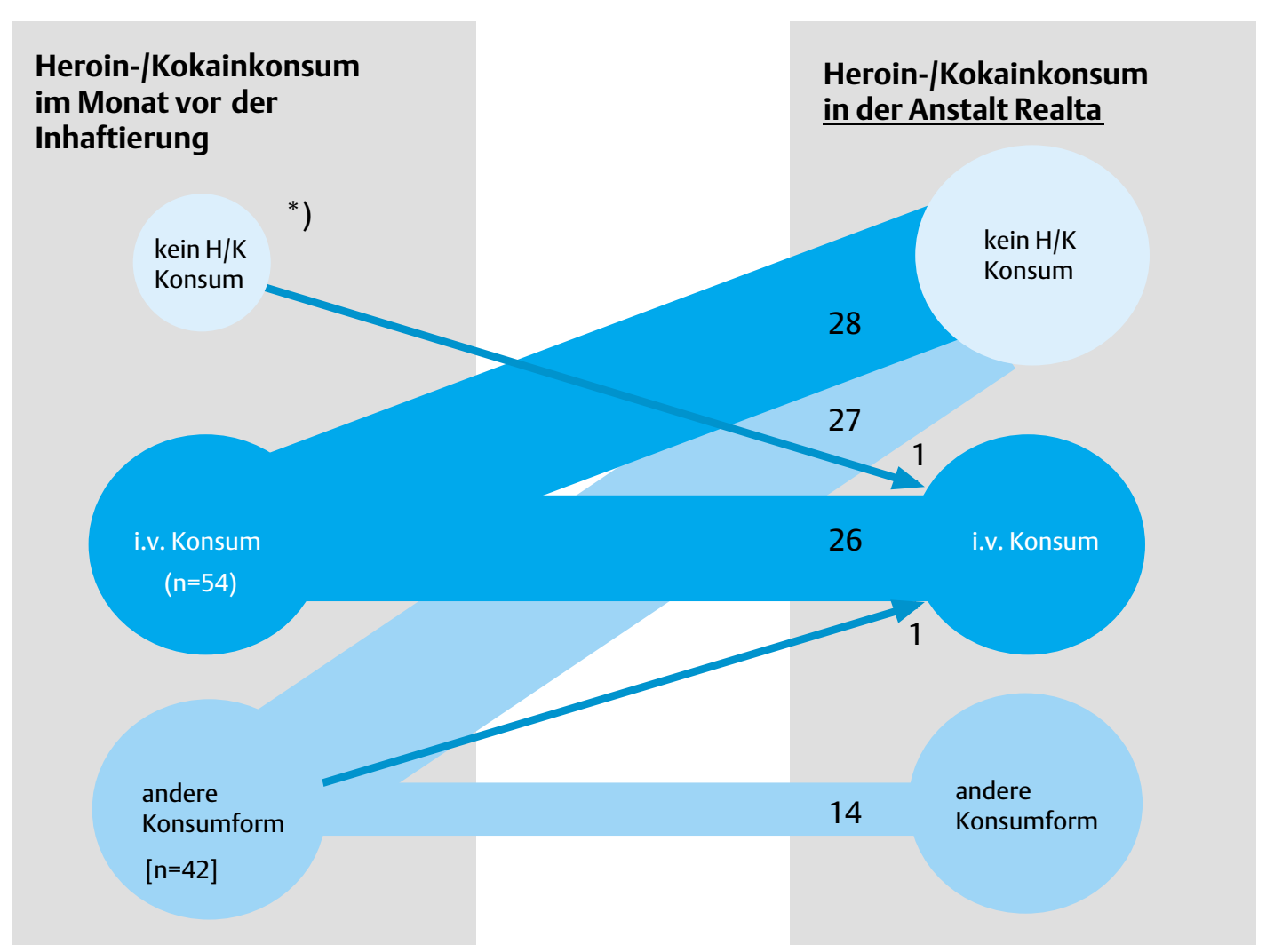

Sanktionen wg. Drogen (Skala links)

andere Sanktionen (Skala links)

- Spritzenverbrauch (Skala rechts)
Abb. 1 Konsum vor und während der Inhaftierung (Gefängnis Realta/Schweiz).

${ }^{*}$ ) Ein Insasse, der i.v.Drogenkonsum jemals im Leben, nicht aber im Monat vor der Inhaftierung angegeben hat, nahm den i.v.-Drogenkonsum im Gefängnis wieder auf.

Abb. 2 Sanktionen und Spritzenverbrauch, Hindelbank, 1994-1995.

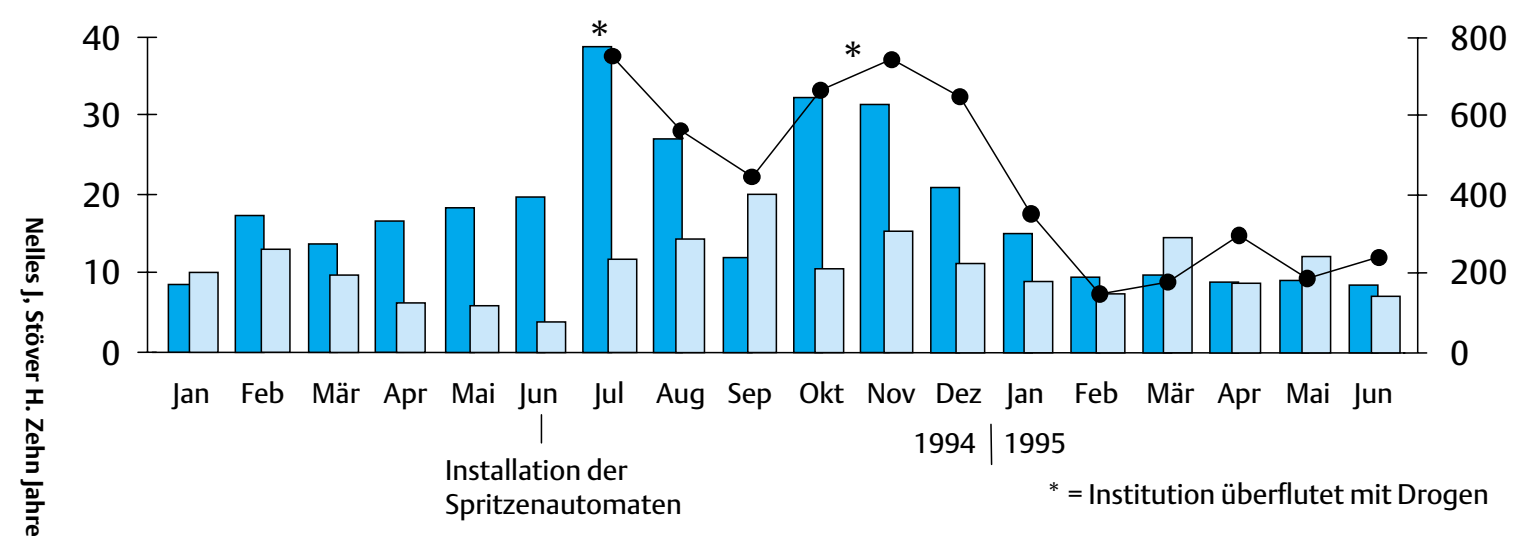

Beteiligten gerechnet werden. In Saxerriet musste so die Begleitevaluation wegen der Widerstände der Insassen und des Personals (Bedenken hinsichtlich zugesicherter Anonymität) bereits vor Ablauf des Projekts beendet werden [16].

Inwieweit schadensbegrenzende Projekte unter Einbezug der Abgabe von sterilen Spritzen von den Häftlingen akzeptiert werden, hängt wesentlich davon ab, in welchem Maße der Zugang zum Spritzenangebot von ihnen als anonym wahrgenommen wird. Beim Spritzenumtausch Anonymität zu gewährleisten ist im Gefängnis zwar schwierig, muss aber dennoch als überaus wichtiges Anliegen wahrgenommen werden. Es bedarf klarer Spielregeln im Verlauf eines Projekts, damit nicht Bedienstete In- sassen, die Spritzen beziehen, unmittelbar auf Drogen kontrollieren und Insassen nicht als Reaktion darauf z.B. Automaten beschädigen und Spritzen in der Anstalt verstreuen (so passiert im Gefängnis Vierlande in Hamburg) [28].

Die Bediensteten akzeptieren den Spritzenumtausch umso mehr, je besser ihnen die Projektziele vermittelt werden, je mehr sie in Planungs- und Entscheidungsprozesse sowie in die Projektvorbereitung und Projektdurchführung einbezogen sind. Gelingt es, auch Gefangene in diesen Prozess einzubeziehen, z.B. Mitwirken von Insassen in einer Projektbegleitgruppe, kann die Akzeptanz potenziert werden. 
Der gemeinsame Gebrauch von Spritzen hat für drogenabhängige Inhaftierte heute keine im Vordergrund stehende rituelle Bedeutung mehr, sondern ist - dort wo Spritzentausch vorkommt - vielmehr die Folge des Mangels an sterilen Spritzen. Die Spritzenabgabe in den untersuchten Gefängnissen hat sich als wirksam dahingehend erwiesen, dass Spritzentausch nicht mehr oder nur noch in Einzelfällen vorkommt oder Spritzentausch auf ohnehin niedrigem Niveau verbleibt. Die außerhalb von Gefängnissen gemachten Erfahrungen ließen sich somit, nicht unerwartet, auch innerhalb von Gefängnissen bestätigen.

In allen Haftanstalten, in welchen diese Aspekte detailliert untersucht wurden, erwies sich der Kenntnisstand zur HIV-Infektion als erfreulich groß; über Hepatitisinfektionen und Hepatitisprophylaxe war der Wissensstand der Inhaftierten (als auch der Bediensteten) äußerst niedrig. Es drängen sich demnach das Bewusstsein sensibilisierende Maßnahmen zur Prophylaxe von Hepatitisinfektionen im Strafvollzug auf, und zwar sowohl Insassen als auch Bedienstete betreffend.

Angesichts der großen Verbreitung von Spritzengebrauch und Drogenkonsum in europäischen Gefängnissen [22] ist es aus schadensbegrenzenden Überlegungen in jeder Strafvollzugsanstalt notwendig, Infektionsrisiken und Schutzmöglichkeiten zu thematisieren, was nicht heißt, dass auch in jeder Anstalt unbedingt Spritzen ausgegeben oder Spritzenautomaten aufgestellt werden müssen. Welche Maßnahmen im Einzelnen zu ergreifen sind oder welche Form der Spritzenabgabe gewählt wird (Handvergabe, Austauschautomat), hängt vom Bedarf, von den Anstaltsstrukturen, den räumlichen Bedingungen der Anstalt und den personellen Kapazitäten sowie von der Drogengebrauchskultur der Gefangenen ab. So ist z. B. der intravenöse Drogenkonsum in Teilen Englands, vor allem aber in den Niederlanden weitaus weniger verbreitet als etwa das Sniffen oder das Rauchen. Demgemäß muss im Vollzug zuerst einmal der Bedarf an sterilen Spritzen erkundet werden, bevor der Ruf nach Spritzenabgabe laut wird, um nicht unter Umständen falsche Signale zu setzen. Werden aber Spritzenautomaten eingesetzt, dann müssen hohe Anforderungen an deren reibungslose Funktion gestellt werden - denn eine gebrauchte Spritze einzugeben und keine neue Spritze zu erhalten, während man im Besitz von Drogen ist, würde den Spritzentausch fördern anstatt ihn zu verhindern.

Es wäre von großem Vorteil, über eine Checkliste zu verfügen, die alle bisherigen Erfahrungen im Vorfeld der Einführung eines Spritzenabgabeprojekts und während der Durchführung des Projekts aufnimmt und daraus resultierende Empfehlungen, Handlungsanweisungen oder Hinweise auf mögliche Schwierigkeiten, aber auch auf optimale Voraussetzungen für zukünftige Projekte verfügbar macht.

Zehn Jahre Spritzenabgabe im Strafvollzug - und die Frage bleibt unbeantwortet, wieso trotz der vielfältigen positiven Erfahrungen aus verschiedenen Projekten die Spritzenabgabe in Strafvollzugseinrichtungen noch immer so umstritten ist und Spritzenabgabe bisher nur in drei europäischen Ländern und auch dort nur in vereinzelten Institutionen zur Infektionsprophylaxe und Schadensbegrenzung im Zusammenhang mit dem Konsum von illegalen Drogen eingeführt worden ist. Die Antwort dürfte nicht im rationalen Bereich zu finden sein, auch wenn die bisherigen
Projekte noch einige Fragen bezüglich der Machbarkeit und Wirksamkeit der Spritzenabgabe z.B. in großen Gefängnissen, in Gefängnissen in nicht industrialisierten Ländern oder in Untersuchungsgefängnissen offen lassen. Grundsätzliche Erfahrungen und Erkenntnisse über Spritzenabgabe im Gefängnis, die eine flächendeckende Einführung dieser Maßnahmen rechtfertigen ließen, gibt es ja in der Zwischenzeit zur Genüge. Spritzenabgabe lässt sich nicht erzwingen. Übergreifende politische Entscheide und Unterstützung sind erforderlich, um der wirksamen Schadensbegrenzung im Strafvollzug zum nötigen Durchbruch zu verhelfen. Wie sehr allgemeine politische und nicht gesundheitspolitische Argumente die Diskussion beeinflussen, zeigt das jüngste Beispiel eines politischen Populismus in Hamburg: Die neue Mitte-Rechts-Koalition vereinbarte in ihrem Koalitionsvertrag vom 19.10.2001: „In den Strafvollzugsanstalten werden zukünftig keine Spritzen mehr ausgegeben. Den Süchtigen werden verstärkt ausstiegsorientierte Hilfen, z.B. verbesserte Therapiemöglichkeiten, angeboten. Dies schließt eine kontrollierte Substitution unter medizinischer Aufsicht ein." Und dies nach durchaus erfolgreichen, mehr als fünfjährigen Bemühungen, wirkungsvolle Modelle der Infektionsprophylaxe zu entwickeln, und ohne dazu zusätzliche Mittel für das anvisierte Kontrollkonzept bereitzustellen [23].

Vielleicht ist die Entwicklung in Spanien, wo alle Gefängnisse per Dekret oberster politischer Instanz angewiesen worden sind, drogenabhängigen Gefangenen sterile Spritzen zur Verfügung zu stellen, geeignet, eine Signalwirkung auch für andere Länder auszulösen. Die Ergebnisse der jeweiligen begleitenden Forschungsprojekte liegen noch nicht vor und werden mit großer Spannung erwartet.

\section{Literatur}

${ }^{1}$ Nelles J. Wie gefährlich sind illegale Drogen? In: Böker W und Nelles J (Hrsg). Drogenpolitik wohin. Bern: Paul Haupt 1992; $181-220$

2 Dolan K. AIDS, drugs and risk behaviour in prison: State of the art. In: Nelles J, Fuhrer A (Hrsg). Harm Reduction in Prison. Bern: Peter Lang 1997; 213-238

${ }^{3}$ Dolan K, Crofts N. A review of risk behaviours, transmission and prevention of blood borne viral infections in Australian prisons. In: Shewan D, Davies B (Hrsg). Drug use and prisons. Amsterdam: Harwood Academic Publishers 2000; 215-232

${ }^{4}$ Ringeling H. Die Suche nach dem rechten Mass. Ethische Erwägungen. In: Böker W, Nelles J (Hrsg). Drogenpolitik wohin. Bern: Paul Haupt 1992; 65-72

${ }^{5}$ Bluthental RN, Kral AH, Gee L, Erringer EA, Edlin BR. The effect of syringe exchange use on high-risk injection drug users: A cohort study. AIDS 2000; 14 (5): $605-611$

${ }^{6}$ Fuhrer A, Nelles J. Harm Reduction in Prison. Aspects of a Scientific Discussion. In: Nelles J, Fuhrer A (Hrsg). Harm Reduction in Prison. Bern: Peter Lang 1997; $13-22$

${ }^{7}$ Shewan D, Davies B. Drug use and prisons. Amsterdam: Harwood Academic Publishers 2000

${ }^{8}$ Nelles J, Waldvogel D, Maurer C, Aebischer C, Fuhrer A, Hirsbrunner HP. Pilotprojekt Drogen- und HIV-Prävention in den Anstalten von Hindelbank. Bundesamt für Gesundheit [EDMZ 311.820D], Evaluationsbericht. Bern 1995

${ }^{9}$ Nelles J, Vincenz I, Fuhrer A, Hirsbrunner HP. Evaluation der HIV- und Hepatitis-Prophylaxe in der Kantonalen Anstalt Realta, Bundesamt für Gesundheit, Evaluationsbericht. Bern 1999

${ }^{10}$ Gore SM, Bird G. HIV, hepatitis and drugs epidemiology in prisons. In: Shewan D, Davies B (Hrsg). Drug use and prisons. Amsterdam: Harwood Academic Publishers 2000; 141 - 172

${ }^{11}$ Nelles J. The contradictory position of HIV-prevention in prison: Swiss experiences. International Journal of Drug Policy 1997; 8: 2-4 
${ }^{12}$ Nelles J, Fuhrer A, Hirsbrunner HP, Harding T. Provision of syringes: The cutting edge of harm reduction strategies in prison? British Medical Journal 1998; 317 (7153): 270-273

${ }^{13}$ Stöver H. Drug and HIV/AIDS Services in European Prisons. Oldenburg: BIS-Verlag 2002

${ }^{14}$ Nelles J, Harding T. Preventing HIV transmission in prison: A tale of medical disobedience and Swiss pragmatism. Lancet 1995; 346 (8989): $1507-1508$

${ }^{15}$ Nelles J, Bernasconi S, Bürki B, Hirsbrunner HP, Maurer C, Waldvogel D. Drogen- und Aids-Prävention im Gefängnis: Pilotprojekt mit freier Spritzenabgabe in den Anstalten Hindelbank bei Bern/Schweiz. In: Stöver H (Hrsg). Infektionsprophylaxe im Strafvollzug. Eine Übersicht über Theorie und Praxis. Berlin: Deutsche AIDS-Hilfe 1994; 101 - 109

${ }^{16}$ Nelles J, Hirsbrunner HP. Infektionsprophylaxe und Drogenprävention in der Strafanstalt Saxerriet. Prozessbegleitung, Anleitung zur anstaltsinternen Datenerhebung und Basisevaluation. Bern: Bundesamt für Gesundheit, Schlussbericht 2002

${ }^{17}$ Koller H. Rechtliche Konsequenzen bei Verweigerung der Abgabe von sterilem Injektionsmaterial in Strafvollzugsanstalten. Bern: Bundesamt für Justiz 1997

${ }^{18}$ Keppler K, Schaper G. Das Spritzenumtauschprogramm in der JVA für Frauen in Vechta/Niedersachsen. In: Jacob J, Keppler, K, Stöver H (Hrsg). LebHaft: Gesundheitsförderung für Drogen Gebrauchende im Strafvollzug. Berlin: Deutsche AIDS-Hilfe 2001; 31 - 34

${ }^{19}$ Lettau S, Sawallisch P, Schulten I, Tieding K, Keppler K, Schaper G. Das Spritzenumtauschprogramm der Justizvollzugsanstalt Lingen. In: Jacob J, Keppler, K, Stöver H (Hrsg). LebHaft: Gesundheitsförderung für Drogen Gerbrauchende im Strafvollzug. Berlin: Deutsche AIDS-Hilfe $2001 ; 35-40$
20 Stöver H. Spritzenumtauschprogramme in drei Hamburger Justizvollzugsanstalten. In: Jacob J, Keppler K, Stöver H (Hrsg). LebHaft: Gesundheitsförderung für Drogen Gerbrauchende im Strafvollzug. Berlin: Deutsche AIDS-Hilfe 2001; 41 - 51

${ }^{21}$ Stark K, Herrmann U, Ehrhardt S, Bienzle U. Modellprojekt Spritzenvergabe im Berliner Justizvollzug. Abschlußbericht der Begleitforschung, Manuskript. Berlin 2001

${ }^{22}$ Rotily M, Weilandt C. European Network on HIV/AIDS and Hepatitis Prevention in Prisons - 3rd Annual Report. Observatoire Régional de la Santé Provence, Alpes Côte d'Azur, Marseille/Wissenschaftliches Institut für die Ärzte Deutschlands. Bonn 1999

${ }^{23}$ Jacob J, Keppler K, Stöver H. LebHaft: Gesundheitsförderung für Drogen Gerbrauchende im Strafvollzug. Berlin: Deutsche AIDS-Hilfe 2001

${ }^{24}$ Monzon IC, Zentralregierung Madrid, persönliche Mitteilung 2002

${ }^{25}$ Laticevski D, Leorda A. HIV-Prävention in Moldawien - Schwerpunkt Spritzenabgabe. In: Jacob J, Keppler K, Stöver H (Hrsg). LebHaft: Gesundheitsförderung für Drogen Gerbrauchende im Strafvollzug. Berlin: Deutsche AIDS-Hilfe 2001; 124-126

${ }^{26}$ Meyenburg R, Stöver H, Jacob J, Pospeschill M. Infektionsprophylaxe im Justizvollzug - Abschlussbericht. Oldenburg: BIS-Verlag 1999

${ }^{27}$ Carrón J. L'implémentation des exchanges de seryngues aux prisons espagnoles. Präsentation an der Konferenz „Encouraging Health Promotion for Drug Users within the Criminal Justice System inc. 4th European Conference of Drug and HIV/AIDS Services in Prison“, 22-25 November 2000. Hamburg

${ }^{28}$ Nelles J, Fuhrer A, Hirsbrunner HP, Harding TW. How does syringe distribution in prison affect consumption of illegal drugs by prisoners? Drug and Alcohol Review 1999; 18: $133-138$ 